\title{
The orthogonal gaseous kinematical decoupling in the Sa spiral NGC 2855
}

\author{
E. M. Corsini ${ }^{1}$, A. Pizzella ${ }^{2}$, and F. Bertola ${ }^{2}$ \\ 1 Osservatorio Astrofisico di Asiago, Dipartimento di Astronomia, Università di Padova, via dell'Osservatorio 8, \\ 36012 Asiago, Italy \\ 2 Dipartimento di Astronomia, Università di Padova, vicolo dell'Osservatorio 2, 35122 Padova, Italy
}

Received 2 September 2001 / Accepted 25 October 2001

\begin{abstract}
We present major and minor-axis kinematics of stars and ionized gas as well as narrow and broad-band surface photometry of the Sa spiral NGC 2855. In the nuclear regions of this unbarred and apparently undisturbed spiral galaxy the gas is rotating perpendicularly to the galaxy disk. We suggest that this kinematically-decoupled component is the signature of an acquisition process in the history of this galaxy.
\end{abstract}

Key words. galaxies: individual: NGC 2855 - galaxies: kinematics and dynamics - galaxies: spiral - galaxies: formation - galaxies: structure

\section{Introduction}

Like fossils, multiple stellar and gaseous components with a misaligned or even opposite angular momentum with respect to the host galaxy conserve the memory of processes driving galaxy formation and evolution. In disk galaxies apart from polar rings they usually are hidden from morphological inspection and are serendipitously discovered by measuring detailed kinematics (see Bertola \& Corsini 1998 for a review). This is the case for a variety of kinematically-decoupled components ranging from the counter-rotating disks of ionized gas observed in a large fraction of S0's (Bertola et al. 1992; Kuijken et al. 1996) as well as in one Sa, NGC 3626 (Ciri et al. 1995; Haynes et al. 2000), to the two counter-rotating stellar disks found in the S0 NGC 4550 (Rubin et al. 1992; Rix et al. 1992), in the early-type spirals NGC 3593 (Bertola et al. 1996), NGC 4138 (Jore et al. 1996; Haynes et al. 2000), and NGC 7217 (Merrifield \& Kuijken 1994), to the orthogonally-rotating stellar cores discovered in two Sa spirals, namely NGC 4698 (Bertola et al. 1999) and NGC 4672 (Sarzi et al. 2000), and to the two

\footnotetext{
Send offprint requests to: E. M. Corsini,

e-mail: corsini@pd.astro.it

* Based on observations carried out at the Observatorio del Roque de los Muchachos, La Palma (Spain) with the Italian Telescopio Nazionale Galileo and at the European Southern Observatory, La Silla (Chile) (ESO 62.N-0463 and 67.B-0230).

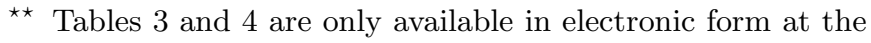
CDS via anonymous ftp to

cdsarc.u-strasbg.fr $(130.79 .128 .5)$ or via

http://cdsweb.u-strasbg.fr/cgi-bin/qcat?J/A+A/382/488
}

counter-rotating gaseous components as in the S0 NGC 7332 (Fisher et al. 1994; Plana \& Boulesteix 1996) and in the Sab NGC 4826 (Braun et al. 1992, 1994; Rubin 1994).

The recent findings by Bettoni et al. (2001) on the higher gas content of counter-rotators and polar ring galaxies with respect to normal galaxies gives further support to the idea that kinematically-decoupled components are the end result of one or more second events that occurred in the history of the host. Yet, even with this insight, the undisturbed appearance with no evident signatures of interaction characterizing most of galaxies with kinematic peculiarities (Bettoni et al. 2001) raises questions about the effective rate of second events and on their role in determining Hubble types. Accretion of external gas and minor mergers seem to be the favoured mechanisms to form decoupled components preserving the preexistent disk (e.g. Thakar \& Ryder 1996; Thakar et al. 1997); nevertheless major mergers can not be ignored since for a narrow range of initial conditions they still represent a viable alternative in building massive counterrotating disks (Pfenniger 1999) and polar ring galaxies (Bekki 1998). Bulge growing, disk heating and spiralpattern smoothing are among the observable consequences of second events in disk galaxies, as predicted by numerical experiments (e.g. Aguerri et al. 2001; Quinn et al. 1993; Thakar \& Ryder 1998). They result in a change of the host morphology toward earlier spiral types.

Recently the presence of a velocity gradient along the minor axis of the disk has been reported in the innermost region of two Sa spirals, namely NGC 4698 (Bertola et al. 1999) and NGC 4672 (Sarzi et al. 2000), characterized 
Table 1. Setup and log of photometric observations.

\begin{tabular}{|c|c|c|}
\hline Parameter & April 18, 1999 & March 27, 2001 \\
\hline Instrument & $\mathrm{TNG}+\mathrm{OIG}$ & ESO $3.6-\mathrm{m}+\mathrm{EFOSC} 2$ \\
\hline CCD & $2 \times \mathrm{EEV} 42-80$ & \#40 Loral/Lesser \\
\hline Pixel size & $13.5 \times 13.5 \mu \mathrm{m}^{2}$ & $15 \times 15 \mu \mathrm{m}^{2}$ \\
\hline Scale & 0.14 pixel $^{-1}$ & 0.31 pixel $^{-1}$ \\
\hline Field of view & $4^{\prime} .9 \times 4^{\prime} .9$ & $5^{\prime} .3 \times 5^{\prime} .3$ \\
\hline Filter & $R$ & $\# 438 \mathrm{H} \alpha^{\mathrm{a}} \quad \# 642 R$ \\
\hline Exposure time & $2 \times 300 \mathrm{~s}$ & $2 \times 600 \mathrm{~s}$ \\
\hline Seeing $F W H M^{\mathrm{b}}$ & $2^{\prime \prime} 1$ & 0.9 \\
\hline
\end{tabular}

${ }^{\text {a }} \lambda_{\mathrm{c}}=6630 \AA, \Delta \lambda_{F W H M}=68 \AA$.

$\mathrm{b}$ Measured on averaged frames.

by an uncommon and remarkable orthogonal geometrical decoupling between bulge and disk. In NGC 4698 the minor-axis velocity gradient is observed in both stars and ionized gas, while in NGC 4672 only in the stellar component. Such a stellar rotation along the disk minor axis indicates the presence of a kinematically isolated core, which is rotating perpendicularly with respect to the disk component. The analysis of the HST images of the nucleus of NGC 4698 shows that in this case the isolated core is a nuclear stellar disk with a scalelength of a few tens of pc (Pizzella et al. 2001). According to Bertola \& Corsini (2000) the presence of these orthogonally-rotating isolated cores indicates that the entire disk of the galaxy could be the end result of the acquisition of external material in polar orbits around a pre-existing oblate spheroid, which became the bulge of the present galaxy. In this scenario the isolated core has been formed by the gaseous material settled down in the symmetry plane of the oblate spheroid during the acquisition process.

In this paper we present a new case of kinematical orthogonal decoupling in the bulge of early-type spiral galaxies which characterizes the gaseous component only. In fact the ionized gas observed in the innermost regions $(|r| \lesssim 0.4 \mathrm{kpc})$ of the Sa NGC 2855 is in orthogonal rotation with respect to the rest of the galaxy.

The paper is organized as follows. We present our photometric and spectroscopic observations of NGC 2855 in Sect. 2 and we analyze its morphological and kinematical properties in Sects. 3 and 4, respectively. Our conclusions are discussed in Sect. 5 .

\section{Observations and data reduction}

\subsection{Narrow and broad-band imaging}

The narrow and broad-band imaging of NGC 2855 were carried out at the Roque de los Muchachos Observatory in La Palma with the Telescopio Nazionale Galileo (TNG) on April 18, 1999 and at the European Southern Observatory (ESO) in La Silla with the 3.6-m ESO telescope on March 27, 2001. The instrumental setup and the observing $\log$ are collected in Table 1.
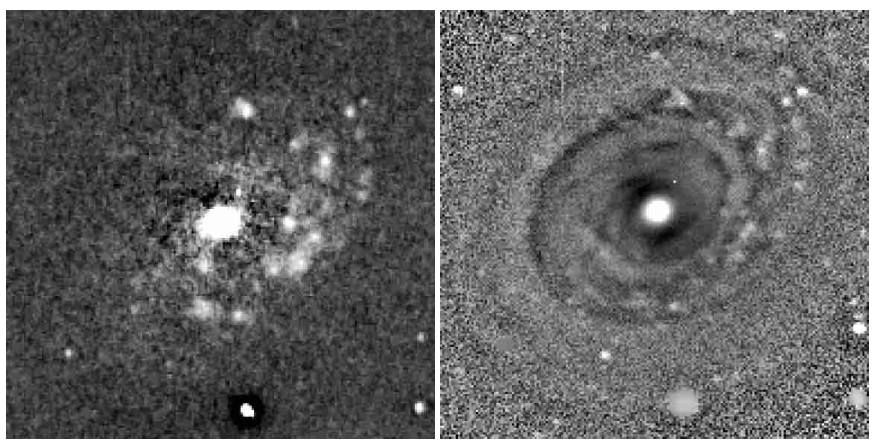

Fig. 1. Continuum-subtracted $\mathrm{H} \alpha+[\mathrm{N}$ II] emission image (left panel) and unsharp masking version of the narrow-band image (right panel) of NGC 2855 unveiling the spiral structure of the galaxy. The size of images is $1^{\prime} \times 1^{\prime}$. North is up and east is left.

Using standard MIDAS ${ }^{1}$ routines the images were bias subtracted, corrected for flatfield using sky flats taken at the beginning and end of each observing night, and cleaned of cosmic rays. The sky background level was removed by fitting a second order polynomial to the regions free of sources in the images. The images obtained with the same filter at the same telescope were shifted and aligned using the common field stars and then they were averaged (after checking the their PSF's were comparable). The map of the $\mathrm{H} \alpha+[\mathrm{N} \mathrm{II}]$ emission was obtained by subtracting the ESO $R$-band image, suitably scaled, from the narrowband image (Fig. 1). To confirm the spiral classification of NGC 2855 we built an unsharp masked image of the galaxy, by dividing its narrow-band frame by itself after being convolved with a circular two-dimensional Gaussian of $\sigma=1^{\prime \prime} .2$ (Fig. 1). This procedure enhanced the spiral pattern of the galaxy as well as any surface-brightness fluctuation and non-circular structure extending over a spatial region comparable with the size of the smoothing Gaussian. Absolute calibration was performed only for the $R$-band images.

We analyzed the isophotal profiles of the galaxy by masking the field stars and fitting ellipses to the isophotes using the IRAF ${ }^{2}$ task ELLIPSE. We first allowed the centers of the ellipses to vary, to test whether the optical disk is disturbed. Within the errors of the fits, we found no evidence of a varying center, therefore the ellipse fits were repeated with the ellipse centers fixed. The resulting surface brightness, ellipticity and position angle radial profiles obtained from both the ESO and TNG $R$-band images are given in Fig. 2.

\subsection{Long-slit spectroscopy}

The spectroscopic observations of NGC 2855 were carried out at the ESO in La Silla with the 1.52-m ESO telescope

\footnotetext{
${ }^{1}$ MIDAS is developed and maintained by the European Southern Observatory.

${ }^{2}$ IRAF is distributed by NOAO, which is operated by AURA Inc., under contract with the National Science Foundation.
} 


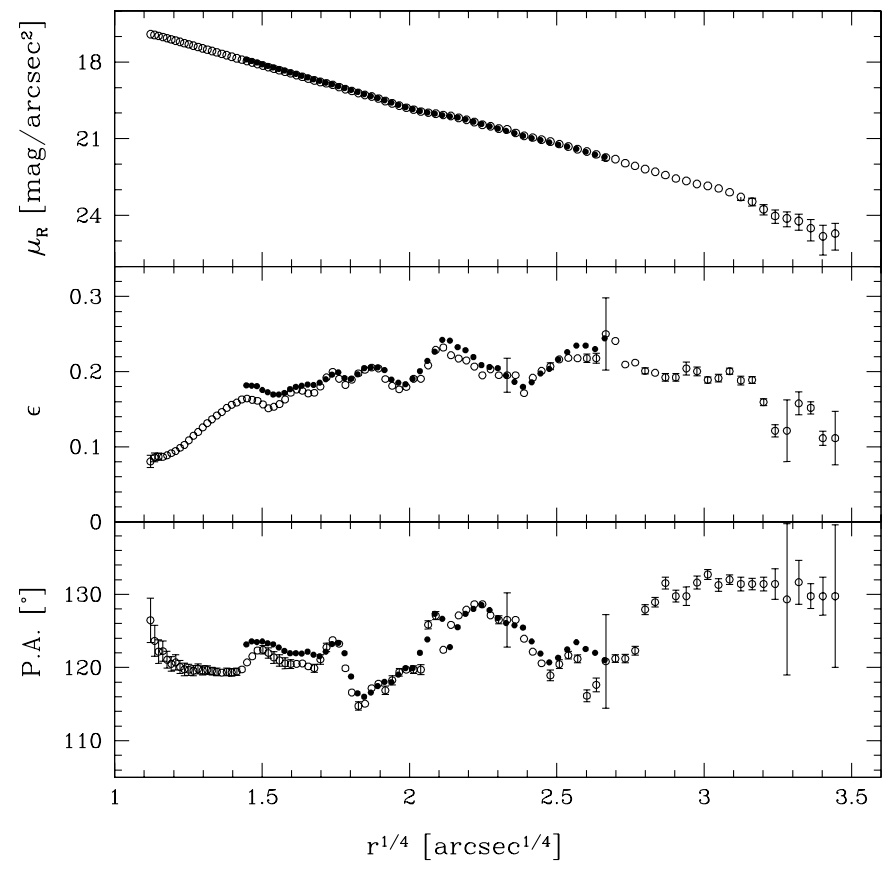

Fig. 2. $R$-band surface-brightness, ellipticity and position angle radial profiles of NGC 2855 measured on the TNG (filled circles) and 3.6-m (open circles) images. Errorbars smaller than symbols are not plotted.

on March 17-22, 1999 and New Technology Telescope (NTT) on May 22, 2001. The details of the instrumental setup and the log of these observing runs are given in Table 2 .

At the beginning of each exposure the galaxy was centered on the slit using the guiding camera. During the March 1999 observing run, we took spectra of several giant stars whose spectral type ranged from G5III to K7III to be used as templates in measuring the stellar kinematics. Comparison spectra were taken before every object exposure. The reduction was carried out with MIDAS. All the spectra were bias subtracted, flatfield corrected, and wavelength calibrated. The contribution of the sky was determined from the outermost $10^{\prime \prime}$ at the two edges of the resulting spectra, where the galaxy light was negligible, and then subtracted. The spectra taken along the same axis in the same run were co-added using the center of the stellar continuum as reference, and cosmic rays were cleaned up in the process.

We measured the stellar kinematics from the galaxy absorption features present in the wavelength range from $5064 \AA$ to $5540 \AA$ and centered on the $\mathrm{Mg}$ line triplet $(\lambda \lambda 5164,5173,5184 \AA)$. We used the Fourier Correlation Quotient method (FCQ, Bender 1990) following the prescriptions of Bender et al. (1994). The spectra were rebinned along the spatial direction to obtain a nearly constant signal-to-noise ratio larger than 20 per resolution element (with a peak of 80 in the innermost regions of the spectra). The galaxy continuum was removed row-by-row by fitting a fourth to sixth order polynomial as in Bender et al. (1994). HR 2035 was adopted as a kinematical
Table 2. Setup and log of spectroscopic observations.

\begin{tabular}{lcc}
\hline Parameter & March 17-22, 1999 & May 22, 2001 \\
\hline Instrument & ESO 1.52-m+B\&C & NTT+EMMI \\
Grating/Grism & $\# 331200 \mathrm{gr} \mathrm{mm}^{-1}$ & $\# 7600 \mathrm{gr} \mathrm{mm}^{-1}$ \\
CCD & $\# 39 \mathrm{Loral} / \mathrm{Lesser}$ & $\# 36 \mathrm{TEK} 2048$ \\
Pixel size & $15 \times 15 \mu \mathrm{m}^{2}$ & $24 \times 24 \mu \mathrm{m}^{2}$ \\
Scale & $0^{\prime \prime} 82 \mathrm{pixel}^{-1}$ & $0^{\prime \prime} 27 \mathrm{pixel}^{-1}$ \\
Dispersion & $0.98 \AA \mathrm{pixel}^{-1}$ & $0.65 \AA \mathrm{pixel}^{-1}$ \\
Slit width & $2^{\prime \prime} .2$ & $1^{\prime \prime} .0$ \\
Wavelength range & $4812-6824 \AA$ & $5630-6968 \AA$ \\
Instrumental $\sigma^{\mathrm{a}}$ & $52 \mathrm{~km} \mathrm{~s}^{-1}$ & $28 \mathrm{~km} \mathrm{~s}^{-1}$ \\
Position angle & $30^{\circ}$ & $30^{\circ}$ \\
Exposure time & $3 \times 60 \mathrm{~min} 2 \times 60 \mathrm{~min}$ & $30 \mathrm{~min}$ \\
Seeing $F W H M$ & $1^{\prime \prime} .5-2^{\prime \prime} .5$ & $0^{\prime \prime} .8$ \\
\hline
\end{tabular}

${ }^{a}$ Measured at $\mathrm{H} \alpha$.

template. This allowed us to derive, for each spectrum, the line-of-sight velocity distribution (LOSVD) along the slit and to measure its moments, namely the radial velocity $v$, the velocity dispersion $\sigma$ and the values of the coefficients $h_{3}$ and $h_{4}$. At each radius, they were derived by fitting the LOSVD with a Gaussian plus third- and fourth-order Gauss-Hermite polynomials $H_{3}$ and $H_{4}$, which describe the asymmetric and symmetric deviations of the LOSVD from a pure Gaussian profile (van der Marel \& Franx 1993; Gerhard 1993). We derived errors on the LOSVD moments from photon statistics and CCD read-out noise, calibrating them by Monte Carlo simulations as done by Bender et al. (1994). These errors do not take into account possible systematic effects due to any template mismatch. The resulting stellar kinematics was reported in Table 3 and plotted in Fig. 4.

The ionized-gas kinematics was measured by the simultaneous Gaussian fit of the emission lines present in the spectra (namely [N $\mathrm{II}] \lambda \lambda 6548,6583, \mathrm{H} \alpha$, and [S II] $\lambda \lambda 6716,6731)$ with an "ad hoc" procedure written within the IDL environment. The galaxy continuum was removed from the spectra as done for the stellar kinematics. We fitted in each row of the continuum-subtracted spectrum a Gaussian to each emission line, assuming them to have the same line-of-sight velocity and velocity dispersion (corrected for heliocentric velocity and instrumental $F W H M$, respectively). An additional absorption Gaussian has been added in the fit to take into account for presence of the $\mathrm{H} \alpha$ absorption line and the flux ratio of the [N II] $\lambda \lambda 6548,6583$ lines have been fixed to 1:3. Far from the galaxy center (for $|r| \gtrsim 10^{\prime \prime}$ ) we averaged adjacent spectral rows in order to increase the signal-to-noise ratio of the relevant emission lines. We calibrated the formal velocity errors obtained from the least-squares fit to match those derived by measuring the rotation curve of nightsky emission lines (cf. Corsini et al. 1999). The resulting ionized-gas kinematics is given in Table 4 and shown in Fig. 4. 


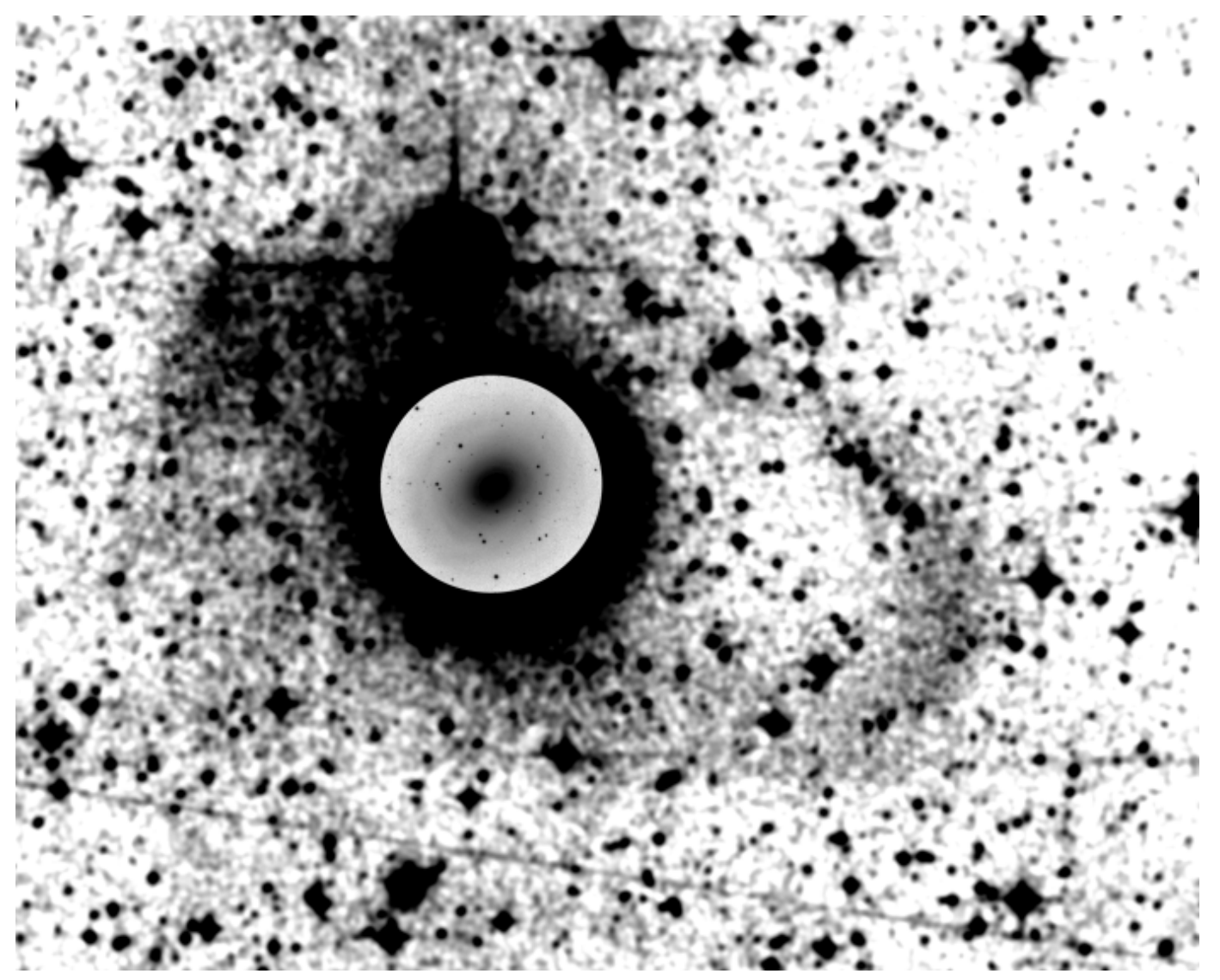

Fig. 3. Deep image of the NGC 2855 field adapted from Malin \& Hadley (1997) and processed to enhance the ring structure surrounding the galaxy. Our ESO $R$-band image is shown in the inset. The frame is $15^{\prime}$ height. North is up and east is left.

\section{Morphology of NGC 2855}

NGC 2855 is an early-type spiral galaxy classified as $\mathrm{Sa}(\mathrm{r})$ by Sandage \& Tammann (1981, RSA hereafter) and RS0/a(rs) by de Vaucouleurs et al. (1991, RC3 hereafter). Its total $B$-band magnitude is $B_{\mathrm{T}}=12.63 \mathrm{mag}$ (RC3) which corresponds to $M_{B}=-19.11 \mathrm{mag}$ for a distance $D=22.3 \mathrm{Mpc}$. We derive the distance from the heliocentric systemic velocity we measured $\left(V_{\odot}=\right.$ $1897 \pm 17 \mathrm{~km} \mathrm{~s}^{-1}$ ) assuming $H_{0}=75 \mathrm{~km} \mathrm{~s}^{-1} \mathrm{Mpc}^{-1}$ and following the precepts of RSA. The measured systemic velocity is in agreement within the errors with the values listed by RSA, Tully (1988) and RC3. The inclination $i=39^{\circ}$ was obtained from the observed axial ratio (whose mean value between $15^{\prime \prime}$ and $30^{\prime \prime}$ is $q=0.79$, Fig. 2) after correcting for the typical intrinsic flattening of S0/a galaxies $\left(q_{0}=0.18\right.$, Guthrie 1992).

Sandage (1961) in The Hubble Atlas of Galaxies considered the galaxy as transition case from $\mathrm{SO}_{3}$ to $\mathrm{Sa}(\mathrm{r})$ due to its circular central absorption ring. Sandage \& Bedke (1994) included NGC 2855 in the section of Carnegie Atlas of Galaxies devoted to Sa galaxies with a spiral pattern entirely defined by dust lanes (Panels 73 and 74). The presence of tightly wound spiral arms in the disk is confirmed by our continuum-subtracted $\mathrm{H} \alpha+[\mathrm{N}$ II] emission image of NGC 2855 and by the unsharp-masked version of its narrow-band image (Fig. 1).

The morphology NGC 2855 appears relatively undisturbed (Rudnick \& Rix 1998) and the galaxy has no close companions (RC3) suggestive of interaction phenomena. However enhanced IIIa-J plates with deep exposures of the NGC 2855 field (Malin \& Hadley 1997) reveal the presence of a structure of low surface brightness $\left(\gtrsim 28 \mathrm{mag} \operatorname{arcsec}^{-2}\right)$, which surrounds the galaxy. We processed this image, which was kindly put at our disposal by D. Malin, to enhance the contrast of the faint structure (Fig. 3). We found that this almost complete loop is elongated in a direction close to the galaxy minor axis $\left(\mathrm{PA} \simeq 60^{\circ}\right)$ and it extends out to about $6^{\prime}$ and $8^{\prime}$ from the galaxy center on its NE and SW side, respectively. It resembles an offset ring with an inclination of about $70^{\circ}$. At fainter levels the stellar disk is nearly face on, confirming a trend already present in the ellipticity profile shown in Fig. 2. Indeed we derive an inclination $i=27^{\circ}$ at $r=141^{\prime \prime}$, corresponding to the outermost observed radius in our ESO $R$-band image. This change of inclination is associated with a variation of the isophotal position angle $\left(\mathrm{PA} \simeq 130^{\circ}\right.$ between $53^{\prime \prime}$ and $141^{\prime \prime}$, and $\mathrm{PA} \simeq 40^{\circ}$ at 2.6 from Fig. 3), and is indicative of a warped stellar disk. The large extension and possible 

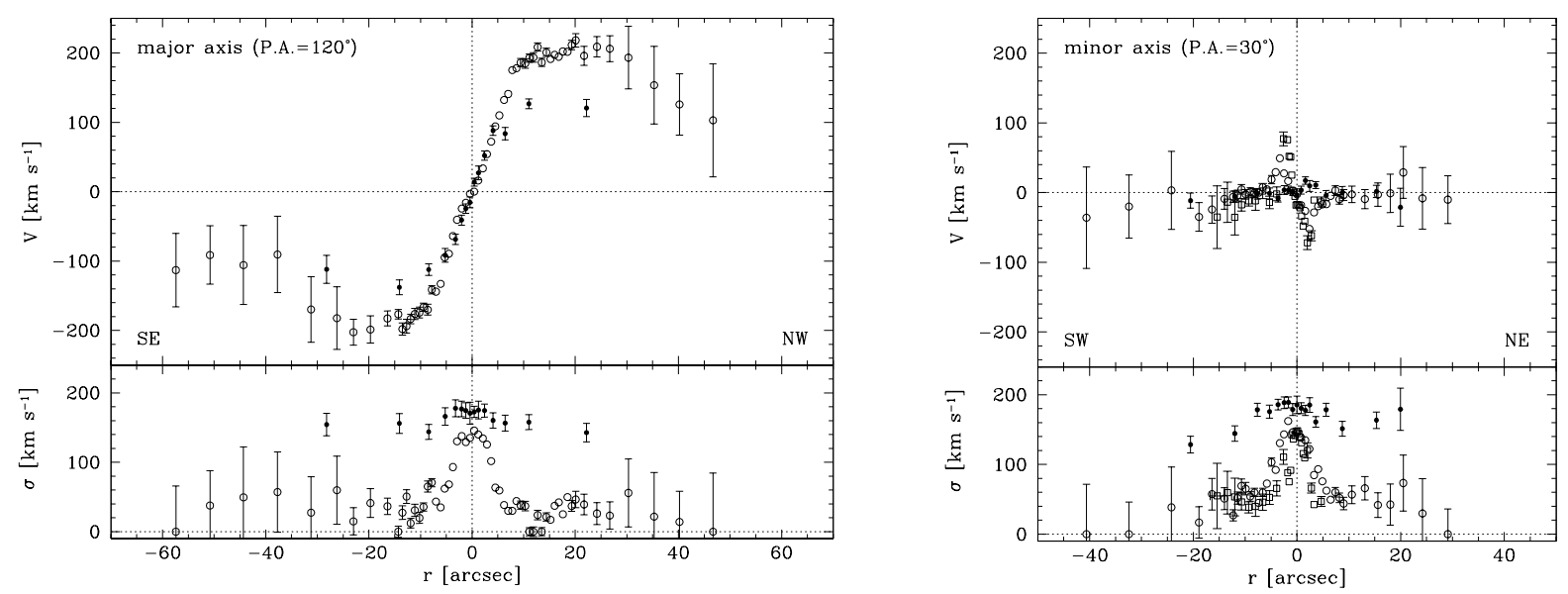

Fig. 4. The stellar (filled circles) and ionized-gas kinematics (open symbols) measured along the major (left panel) and minor axis (right panel) of NGC 2855. All velocities are plotted as observed after subtracting the value of the systemic heliocentric velocity and without applying any inclination correction. Errorbars smaller than symbols are not plotted. In the lower panel open circles and open squares represent data obtained with the ESO 1.52-m telescope and NTT, respectively.

warp of the disk component could explain the discrepancy between the available bulge-disk decompositions of the surface brightness of NGC 2855. Indeed the disk results are embedded in the bulge at all the observed radii according to the $V$ (Baggett et al. 1998) and $K$-band photometric decompositions (Möllenhoff \& Heidt 2001). On the other hand, the disk dominates the galaxy light for $r \gtrsim 35^{\prime \prime}$ in the $B$ and $J$-band photometric decomposition performed by Boroson (1981) and Möllenhoff \& Heidt (2001), respectively.

\section{Kinematics of NGC 2855}

The velocity curves and velocity dispersion profiles we measured for the stellar and gaseous components along the major and minor axis of NGC 2855 are presented in Fig. 4.

The stellar kinematics does not show any peculiarity on both the observed axes. It extends out to $28^{\prime \prime}$ (corresponding to $3.0 \mathrm{kpc}$ ) and $20^{\prime \prime}$ from the center (corresponding to $2.8 \mathrm{kpc}$ after correcting for inclination) along the major and minor axis, respectively. The velocity dispersion declines from a central value of about 190 to $150 \mathrm{~km} \mathrm{~s}^{-1}$. Along the major axis we observe a maximum $\Delta V \simeq 260 \mathrm{~km} \mathrm{~s}^{-1}$ at $|r| \approx 12^{\prime \prime}$. Along the minor axis there is no significant stellar velocity gradient and the velocity dispersion ranges from a maximum of about 190 to $130 \mathrm{~km} \mathrm{~s}^{-1}$ at the farthest measured radius.

The major-axis kinematics of the ionized gas is measured out to $57^{\prime \prime}(6.2 \mathrm{kpc})$ from the nucleus. Gas rotates slower than stars in the inner $|r| \lesssim 4^{\prime \prime}(0.4 \mathrm{kpc})$. The opposite is true further out. Ionized gas reaches its maximum $\Delta V \simeq 420 \mathrm{~km} \mathrm{~s}^{-1}$ at $|r| \approx 20^{\prime \prime}$. At larger radii the gas rotation velocity decreases to about $100 \mathrm{~km} \mathrm{~s}^{-1}$ on both sides of the nucleus. We interpret this velocity trend as the result of a projection effect rather an intrinsic phenomenon and therefore we conclude that the gaseous as well as the stellar one tends to be more face-on at larger radii. The gas velocity dispersion peaks at a central value of about $150 \mathrm{~km} \mathrm{~s}^{-1}$, and outwards it decreases, remaining lower than $60 \mathrm{~km} \mathrm{~s}^{-1}$ for $|r| \gtrsim 10^{\prime \prime}$. We derived the minor-axis kinematics of the gaseous component out to $11^{\prime \prime}(1.5 \mathrm{kpc}$ after deprojecting) and to $41^{\prime \prime}$ (5.7 kpc after deprojecting) from the NTT and ESO 1.52-m spectrum, respectively. In the NTT spectrum the velocity curve shows a steep gradient in the nucleus rising to a maximum observed rotation of about $75 \mathrm{~km} \mathrm{~s}^{-1}$ at $|r| \approx 2^{\prime \prime}$. At larger radii the gas velocity drops to zero and no rotation is detected for $|r| \gtrsim 6^{\prime \prime}$. The presence of a sharp rotation of the gas component along the galaxy minor axis is confirmed by the kinematics obtained from the ESO 1.52-m spectrum, although these data are characterized by a lower spectral and spatial resolution with respect to the NTT ones. The gas velocity dispersion along the minor axis shows the same behaviour observed along the major one.

\section{Discussion and conclusions}

The analysis of the velocity curves of stars and ionized gas along both the major and minor axis of NGC 2855 shows a kinematical decoupling between the gas in the innermost regions of the galaxy and the remaining gas. Indeed the innermost gas shows a central steep velocity gradient along the minor axis (for $|r| \lesssim 2^{\prime \prime}$ ) where stars do not have any significant rotation. The gas rotates slightly slower than the stars along the galaxy major axis (for $|r| \lesssim 4^{\prime \prime}$ ). On the contrary, the outer gas rotates faster than the stars along the major axis and shows no rotation along the galaxy minor axis.

We exclude that the gas velocity gradient we measure along the galaxy minor axis is due to non-circular motions in a barred potential. Indeed we do not observe any bar structure in low-inclined disk of the galaxy either on our $R$-band image or in the near-infrared images of 
Peletier et al. (1999) and Möllenhoff \& Heidt (2001). In addition, the radial profiles of ellipticity and position angle do not show the signatures of the presence of a nuclear bar, as discussed by Wozniak et al. (1995).

Since the minor-axis gas rotation is measured inside the bulge-dominated region and since the intrinsic shape of bulges is generally triaxial (Bertola et al. 1991) we can consider the kinematical decoupling observed in NGC 2855 as an effect due to the bulge triaxiality. If the gas component is in one of the equilibrium planes of the bulge (as in the case of the Sa NGC 4845 studied by Bertola et al. 1989; Gerhard et al. 1989) the sharp rise and fall of the gas rotation velocity observed for $|r| \lesssim 6^{\prime \prime}$ along the minor axis might be caused by strong non-circular motions. However this is unlikely, since we expect a mild triaxiality for the bulge of NGC 2855 because of the lack of a significant isophotal twisting for $r \lesssim 10^{\prime \prime}$ (Fig. 2). We therefore interpret the observed ionized-gas velocity field as being due to the presence of two kinematicallydecoupled gaseous components, which are rotating around two roughly orthogonal axes. They are the shortest and the longest axes of the triaxial bulge. In this picture the innermost gas is moving onto the equilibrium plane orthogonal to the equatorial one, where both the galaxy disk and outer gas component are settled. If this is the case, the kinematical decoupling is also consistent with an abrupt inner warp of the ionized-gas component.

Kinematical decoupling between two components of a galaxy suggests the occurrence of a second event, so it is easy to explain the existence of the innermost orthogonally-rotating gas as being due to external material acquired from a direction close to the bulge equilibrium plane orthogonal to that of the galaxy disk. This scenario is supported by the presence of the faint structure which surrounds the galaxy and is elongated in a direction close to the galaxy minor axis. Part of the gaseous material associated with this loop could be sinked towards the galaxy nucleus sweeping away the gas of the disk and giving rise to the decoupled component.

Although it involves a second event, the mechanism of formation of the kinematically-decoupled component in NGC 2855 seems to be different from that of NGC 4698 and NGC 4672, where the entire disk could be formed in the acquisition process (Bertola \& Corsini 2000). In fact it has to be pointed out that in NGC 2855 the innermost gas has not yet turned into stars, while it is partially and completely transformed into stars in NGC 4698 and NGC 4672, respectively. According to this result, although the low inclination of NGC 2855 does not preclude that its bulge could be an oblate spheroid protruding out of the disk plane but we have to explain why star formation did not occur in the inner orthogonally-rotating gaseous component. Such a kinematically-decoupled gas may represent an early stage in the formation of an orthogonally-rotating stellar core.

The case of NGC 2855 gives further support to the mounting evidence that in nearby galaxies even with an apparently undisturbed morphology we find the signatures of on-going or recent acquisition phenomena if we study them in detail. A better knowledge of second events in present-day galaxies is a key to understanding such processes in the early universe, when they were more frequent due to the higher galaxy density.

Acknowledgements. We acknowledge Ralf-Jürgen Dettmar for the ESO 3.6-m narrow-band imaging of NGC 2855. We are indebted to R. Bender and R. Saglia for providing us with the FCQ package we used for measuring the stellar kinematics. We are also grateful to D. Malin for the image of NGC 2855 we used in Fig. 3.

\section{References}

Aguerri, J. A. L., Balcells, M., \& Peletier, R. F. 2001, A\&A, 367,428

Baggett, W. E., Baggett, S. M., \& Anderson, K. S. J. 1998, AJ, 116, 1626

Bekki, K. 1998, ApJ, 502, L133

Bender, R. 1990, A\&A, 229, 441

Bender, R., Saglia, R. P., \& Gerhard, O. E. 1994, MNRAS, 269, 785

Bertola, F., \& Corsini, E. M. 1998, in Galaxy Interactions at Low and High Redshift, ed. J. Barnes, \& D. B. Sanders (Kluwer, Dordrecht), IAU Symp., 186, 149

Bertola, F., \& Corsini, E. M. 2000, in Galaxy Dynamics: from the Early Universe to the Present, ed. F. Combes, G. A. Mamon, \& V. Charmandaris (ASP, San Francisco), ASP Conf. Ser., 197, 115

Bertola, F., Buson, L. M., \& Zeilinger, W. W. 1992, ApJ, 401, L79

Bertola, F., Rubin, V. C., \& Zeilinger 1989, ApJ, 345, L29

Bertola, F., Vietri, M., \& Zeilinger, W. W. 1991, ApJ, 374, L13

Bertola, F., Cinzano, P., Corsini, E. M., et al. 1996, ApJ, 458, L67

Bertola, F., Corsini, E. M., Vega Beltrán, J. C., et al. 1999, ApJ, 519, L17

Bettoni, D., Galletta, G., \& Prada, F. 2001, A\&A, 374, 83

Bettoni, D., Galletta, G., García-Burillo, S., \& RodríguezFranco, A. 2001, A\&A, 374, 421

Boroson, T. 1981, ApJS, 46, 177

Braun, R., Walterbos, R. A. M., \& Kennicutt, R. C. 1992, Nature, 360, 442

Braun, R., Walterbos, R. A. M., Kennicutt, R. C., \& Tacconi, L. J. 1994, ApJ, 420, 558

Ciri, R., Bettoni, D., \& Galletta, G. 1995, Nature, 375, 661

Corsini, E. M., Pizzella, A., Sarzi, M., et al. 1999, A\&A, 342, 671

de Vaucouleurs, G., de Vaucouleurs, A., Corwin, H. G. Jr., et al. 1991, Third Reference Catalogue of Bright Galaxies (Springer-Verlag, New York) (RC3)

Fisher, D., Illingworth, G., \& Franx, M. 1994, AJ, 107, 160

Gerhard, O. E. 1993, MNRAS, 265, 213

Gerhard, O. E., Vietri, M., \& Kent, S. M. 1989, ApJ, 345, L33

Guthrie, B. N. G. 1992, A\&AS, 93, 255

Haynes, M. P., Jore, K. P., Barrett, E. A., Broeils, A. H., \& Murray, B. M. 2000, AJ, 120, 703

Jore, K. P., Broeils, A. H., \& Haynes, M. P. 1996, AJ, 112, 438

Kuijken, K., Fisher, D., \& Merrifield, M. R. 1996, MNRAS, 283,543 
Malin, D., \& Hadley, B. 1997, PASA, 14, 52

Merrifield, M. R., \& Kuijken, K. 1994, ApJ, 432, 575

Möllenhoff, C., \& Heidt, J. 2001, A\&A, 368, 16

Peletier, R. F., Knapen, J. H., Shlosman, I., et al. 1999, ApJS, 125,363

Pfenniger, D. 1999, in Galaxy Interactions at Low and High Redshift, IAU Symp. 186, ed. J. Barnes, \& D. B. Sanders (Kluwer, Dordrecht), 157

Pizzella, A., Corsini, E. M., Morelli, L., et al. 2001, ApJ, submitted

Plana, H., \& Boulesteix, J. 1996, A\&A, 307, 391

Quinn, P. J., Hernquist, L., \& Fullagar, D. P. 1993, ApJ, 403, 74

Rix, H., Franx, M., Fisher, D., \& Illingworth, G. 1992, ApJ, 400, L5

Rubin, V. C. 1994, AJ, 107, 173

Rubin, V. C., Graham, J. A., \& Kenney, J. D. P. 1992, ApJ, 394, L9

Rudnick, G., \& Rix, H.-W. 1998, AJ, 116, 1163
Sandage, A. 1961, The Hubble Atlas of Galaxies (Carnegie Institution, Washington)

Sandage, A., \& Bedke, J. 1994, The Carnegie Atlas of Galaxies (Carnegie Institution and Flintridge Foundation, Washington) (RSA)

Sandage, A., \& Tammann, G. A. 1981, A Revised ShapleyAmes Catalog of Bright Galaxies (Carnegie Institution, Washington) (CAG)

Sarzi, M., Corsini, E. M., Pizzella, A., et al. 2000, A\&A, 360, 439

Thakar, A. R., \& Ryden, B. S. 1996, ApJ, 461, 55

Thakar, A. R., \& Ryden, B. S. 1998, ApJ, 506, 93

Thakar, A. R., Ryden, B. S., Jore, K. P., \& Broeils, A. H. 1997, ApJ, 479, 702

Tully, R. B. 1988, Nearby Galaxy Catalog (Cambridge University Press, Cambridge)

van der Marel, R. P., \& Franx, M. 1993, ApJ, 407, 525

Wozniak, H., Friedli, D., Martinet, L., Martin, P., \& Bratschi, P. 1995, A\&AS, 111, 115 